ISSN 1814-1196

Научный вестник НГТУ

том 75, № 2, 2019, с. 101-114

ИНФОРМАТИКА, ВЫЧИСЛИТЕЛЬНАЯ ТЕХНИКА

И УПРАВЛЕНИЕ http://journals.nstu.ru/vestnik

Science Bulletin of the NSTU

Vol. 75, No. 2, 2019, pp. 101-114

\author{
INFORMATICS, \\ COMPPUTER ENGINEERING \\ AND CONTROL
}

УдК 510.6., 004.421, 523.21

DOI: 10.17212/1814-1196-2019-2-101-114

\title{
Комплементарные отношения
}

\section{В.Я. ЦВЕТКОВ}

109029, РФ, г. Москва, ул. Нижегородская, 27, строение 1, Научно-исследовательский и проектно-конструкторский институт информатизации, автоматизации и связи на железнодорожном транспорте (НИИАС)

cvj2@mail.ru

При организации вычислений вычислительные процессы должны обладать свойствами согласованности и непротиворечивости. Эти свойства можно рассматривать как результат комплементарности и наличия комплементарных отношений. В теории системы сложные системы должны обладать свойствами целостности и полноты. Наличие этих свойств есть признак комплементарных отношений. В науках о Земле, в частности в области геоинформатики, используют набор последовательных технологий обработки пространственной информации. Обработке информации предшествуют технологии сбора информации. За ними следуют технологии первичной обработки и отбраковки. Затем идут технологии моделирования, хранения и так далее. Совокупность разных технологий, решающих общую задачу, связывают комплементарные отношения. Статья исследует комплементарные отношения как специфический вид сложных отношений. Сложность заключается в многообразии форм комплементарных отношений. Комплементарные отношения могут присутствовать в коммуникационных сетях, ситуациях, в сложных системах. Комплементарные отношения создают свойство комплементарности или состояние комплементарности. Статья систематизирует виды комплементарности и вводит понятия: функциональная комплементарность, системная комплементарность, логическая комплементарность. Логическая комплементарность дополняет другие виды комплементарности. Она позволяет проверять различные технологии и структуры на непротиворечивость и истинность. Логическая комплементарность позволяет проверять также различные процессы на истинность и непротиворечивость. Многие методы верификации программ используют свойство комплементарности неявно.

Ключевые слова: комплементарность, отношения, информационные отношения, комплементарные отношения, непротиворечивость

\section{ВВЕДЕНИЕ}

Комплементарность является полисемическим понятием [1]. Лингвистически она является аналогом дополнительности и может рассматриваться как качество, свойство, отношение, состояние. Анализ определений и трактовок

\footnotetext{
* Статья получена 13 декабря 2018 г.
} 
показывает, что большая часть этого понятия отражается прямыми свойствами: согласованность, дополняемость, сопряженность, следуемость, ситуативность. Понятие комплементарности отражается также оппозиционными признаками - непротиворечивостью и неоппозиционностью.

Оксфордский словарь [2] дает предикативную интерпретацию комплементарности: отношение или ситуащия, в которой вещи улучшают или подчеркивают качества друг друга. Словарь Мериам-Вебстер [3] содержит атрибутивную интерпретацию: комплементарность - это качество или состояние (взаимодополняемости). В словаре делового английского языка это понятие трактуется как принции дополнительности. Анализ работ в области комплементарности дает основание утверждать, что результатом комплементарности является согласованность, упорядоченность, системность, эмерджентность и в отдельных случаях синергия. В силу этого комплементарность выступает как оппозиция хаоса и неопределенности. Комплементарность применяют в медицине [4], биологии [5, 6], инвестиционной деятельности $[7,8]$ и образовании [9]. Комплементарный подход применяют при оценке рынка $[9,10]$. Комплементарность как качество всегда вызывает улучшение свойств ресурсов [11], совокупностей и систем.

Развитию теории комплементарности способствовали области математики, включающие оптимизацию [1], нелинейный анализ, проблемы равновесия, теорию вариационного неравенства, теорию неподвижных точек, теорию топологических степеней [12]. Теория комплементарности интенсивно развивается в психологии [13] и математике [14].

В теории математической оптимизации проблема линейной комплементарности часто возникает в вычислительной механике и включает в себя квадратичное программирование. Проблемы комплементарности были первоначально изучены, в линейном программировании и квадратичном программировании они представляют собой проблему линейной комплементарности (LCP), проблему нелинейной комплементарности (NCP) и проблему смешанной комплементарности (MCP).

В математике проблема комплементарности представляет собой частную проблему оптимизации. Задача оптимизации функции двух векторных переменных связана с определенными требованиями, которые включают требование того, что скалярное произведение двух векторов должно быть равно нулю [15], т. е. они должны быть ортогональны.

Для конечномерных вещественных векторных пространств комплементарность означает, что если есть векторы $X$ и $Y$ со всеми неотрицательными компонентами ( $x_{i} \geq 0$ и $y_{i} \geq 0$ для всех $\left.i\right)$, то для каждой пары компонент $\left(x_{i}, y_{i}\right)$ одна из пары компонент должна быть равна нулю, что обеспечивало комплементарность как дополняемость. Например, $X=(1,0)$ и $Y=(0,2)$ дополняют друг друга - они комплементарны. Но $X=(1,1)$ и $Y=(2,0)-$ не комплементарны. Проблема комплементарности является частным случаем вариационного неравенства. Несмотря на изученность в математике и психологии, можно констатировать недостаточное исследование этого феномена как отношения. Это делает актуальным исследование комплементарности и эффектов, которые она создает. В статье рассматривается комплементарность как специфическое отношение. 


\section{1. ПОСТАНОВКА ЗАДАЧИ}

Принципиальным является то, что комплементарность не может быть описана формально одним типом отношений. Она охватывает комплекс разных отношений: логических, функциональных, теоретико-множественных, лингвистических, математических, юридических. При рассмотрении комплементарности обязательно рассматривают не менее двух объектов. В исчислении высказываний $[16,17]$ комплементарность выражается отношениями импликации, эквивалентности и конъюнкции. Объекты $A$ и $B$ комплементарны, если между ними имеют место отношения, задаваемые комплементарным рядом:

$$
A \rightarrow B \equiv 1 ; A \sim B \equiv 1 ; A \wedge B \equiv 1 ; A \mid B \equiv 0 ; A \vdash B .
$$

Выражение (1) является примером комплементарного ряда и описывает следующие отношения между $A$ и $B$ : импликация (истина), эквивалентность (истина), коньюнкция (истина), | - штрих Шеффера (ложь), Объекты $A$ и $B$ не комплементарны, если между ними имеют место другие отношения, которые дает пример некомплементарного ряда.

$$
A \rightarrow B \equiv 0 ; A \oplus B \equiv 1 ; A \wedge B \equiv 0 ; A \vee B \equiv 0 .
$$

Выражение (2) является примером некомплементарного ряда и описывает следующие отношения между $A$ и $B$ : импликация (ложь), «исключающее или» (истина), конъюнкция (ложь), дизъюнкция (ложь) и т. д.

Принципиальным для выражений (1) и (2) является то, что один и тот же логический оператор может описывать комплементарность или некомплементарность в зависимости от логического значения. Это подчеркивает сложность описания комплементарности.

В исчислении предикатов комплементарность как отношение отображается набором двухместных предикатов. Можно ввести двуместный предикат $R(x, y)$, который отображает комплементарность между $(x, y)$.

Возможна множественная комплементарность между несколькими объектами. Примером является гипотеза в математической логике. Напомним, что гипотезой $B$ формулы $A$ понимается такая формула $B$, что

$$
(B \rightarrow A) \equiv 1 .
$$

Гипотеза $B(x)$ формулы $A$ называется простой, если она есть конъюнкция переменных или их отрицаний и после отбрасывания любого из ее сомножителей перестает быть гипотезой формулы $A$. Комплементарными являются конъюнкты гипотезы. В качестве примера рассмотрим 9 конъюнктов:

$$
B(x)=(\mathrm{x} 1 \wedge \mathrm{x} 2 \wedge \mathrm{x} 3 \wedge \mathrm{x} 4 \wedge \neg \mathrm{x} 5 \wedge \neg \mathrm{x} 6 \wedge \neg \mathrm{x} 7 \wedge \mathrm{x} 8 \wedge \mathrm{x} 9) .
$$


Выражение (3) является комплементарным. Некомплементарным является выражение (4), связанное с выражением (3). Оно получается исключением одного из переменных $(\neg \mathrm{x} 6)$ :

$$
\bar{B}=(\mathrm{x} 1 \wedge \mathrm{x} 2 \wedge \mathrm{x} 3 \wedge \mathrm{x} 4 \wedge \neg \mathrm{x} 5 \wedge \neg \mathrm{x} 7 \wedge \mathrm{x} 8 \wedge \mathrm{x} 9) .
$$

В выражении (4) величина $\bar{B}$ обозначает «не гипотезу», т. е. «не фактор». Выражений типа (2) может быть много, выражение (3) только одно. Некомплементарных ситуаций или выражений всегда больше, чем комплементарных. Это определяет технику организации комплементарности. Согласованность осуществляют множеством методов, что затрудняет выработку единого подхода формирования комплементарности. Непротиворечивость логическая категория, которая может быть также реализована средствами математической логики для формирования комплементарности. Непротиворечивость может служить инструментом формирования комплементарности. В случае анализа непротиворечивости можно выработать общий подход к оценке и созданию комплементарности средствами математической логики, например, на основе метода резолюций. Инверсия комплементарного выражения $\bar{B}$ или $\neg B$ всегда означает некомплементарность.

Комплементарность выражается не только средствами двоичной логики, но средствами троичной логики. Известное правило переноса транзитивности отражает комплементарность отношений между тремя объектами. «Если из $A$ следует $B$, а из $B$ следует $C$, то из $A$ следует $C$ ».

Пример. Площадь круга $A$ больше площади прямоугольника $B$. Площадь прямоугольника $B$ больше площади треугольника $C$. Следовательно, площадь $A$ больше площади $C$. Это правило действительно в математике. В реальной жизни оно может нарушаться [18]. Это часто бывает на спортивных соревнованиях: команда $A$ побеждает команду $B$. Команда В побеждает команду $C$, но команда $C$ побеждает команду $A$. В этом случае имеет место некомплементарность. Геометрически эти ситуации отражают треугольники согласования и противоречия.
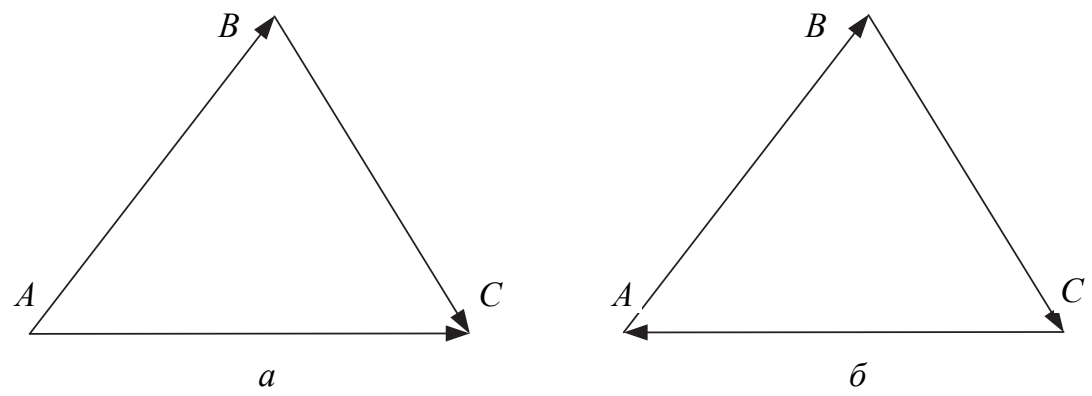

Puc. 1. Треугольники согласования (комплементарности) (a) и противоречия (некомплементарности) (б)

Fig. 1. Triangles reconciliation (complementarity) (a) and contradictions (non-complementarity) $(b)$ 
Отношения на рис. 1 показывают комплементарную и некомплементарную ситуацию. Это говорит о том, что в отдельных случаях комплементарность или некомплементарность выражаются графическими или топологическими схемами. В частности, ациклические алгоритмы [19] являются примером комплементарности. Ситуации зацикливания или зависания характеризуют некомплементарность.

\section{2. КОМПЛЕМЕНТАРНОСТЬ КАК СВОЙСТВО ИЛИ СОСТОЯНИЕ ИНФОРМАЦИОННОЙ СИТУАЦИИ}

Комплементарность является не индивидуальной, а множественной характеристикой. Поэтому ее легко применить в модели информационной ситуации, которая описывает множество объектов и отношений. Комплементарность как отношение и состояние можно оценить для информационной ситуации. В сложной системе параметры комплементарны относительно общей задачи системы. В информационной ситуации такой задачи первоначально нет. Информационная ситуация $[20,21]$ является универсальной информационной моделью, описывающей реальную ситуацию. Ее начальная главная функция - описание реальной ситуации с выделенными в ней объектами, отношениями и связями. Информационная ситуация может быть рассмотрена как множественное условие, которое используют при решении разных задач. При появлении конкретной задачи в информационной ситуации отбирают те параметры или показатели, которые необходимы для решения именно этой задачи. Выделенные параметры называют ключевыми параметрами или ключевыми показателями. В этом случае информационная ситуация преобразуется в специализированную модель, которая необходима для решения задачи или для принятия конкретного решения. В специализированной информационной ситуации можно оценивать комплементарность применительно к решаемой задачи.

Из (15) и (17) следует, что задача управления триподом (см. рис. 1) может быть решена путем применения трех практически аналогичных агентов, каждый из которых решает задачу управления одной из систем второго порядка (17). Формально решения этих систем не зависят друг от друга, однако их практическая реализация должна осуществляться с учетом совместных соотношений (11), (16) и ограничений (6), (10).

Комплементарность информационной ситуации упрощает решение задачи. Некомплементарность параметров информационной ситуации затрудняет решение задачи или повышает сложность решения задачи. В информационной ситуации комплементарность между параметрами, процессами или объектами схожа с понятием информационное соответствие [22, 23]. Информационное соответствие всегда выражается отношением эквивалентности или изоморфизмом. Комплементарность выражается отношениями эквивалентности, принадлежности, импликации, конъюнкции и другими операторами. Принципиально то, что она может соответствовать одному из названных отношений или включать их в комбинации. Это означает, что комплементар- 
ность может описываться не только простой логической связкой, но и логической формулой. Комплементарность ситуации можно оценить как комплементарность по одному параметру, по нескольким параметрам или по всем ключевым параметрам данной информационной ситуации. Это приводит к понятию частичной или полной комплементарности информационной ситуации.

\section{3. КОМПЛЕМЕНТАРНОСТЬ МЕЖДУ СИСТЕМАМИ}

Комплементарность внутри системы присутствует всегда. Она может быть сильной или слабой, но имеет место. Комплементарность между системами может быть или не быть. Комплементарность между системами можно оценить исходя из системного описания. Системный подход $[24,25]$ позволяет описать сложную систему (SYS) с выделением ее ключевых показателей. Применительно к задаче исследования комплементарности можно дать следующее описание системы:

$$
S Y S=<F, \text { Str, E, C, R, G, int, out, Sem, Seq, D>. }
$$

В выражении (5) $F$ - множество функций системы; Str - структура системы; $E$ - множество элементов в системе; $C$ - множество связей; $R-$ множество отношений; $G$ - множество целей; int - множество входов; out - множество выходов системы; Sem - семантическое содержание системы или используемых данных; $\mathrm{Seq}$ - прескриптивная модель действий системы; $D$ типы данных, используемых системой. Практически на всех выделенных параметрах сложной системы комплементарность может быть или не быть. Комплементарность может быть внутренней между частями системы. Комплементарность может быть внешней между разными системами. Наличие внутренней комплементарности улучшает эффективность системы, отсутствие - ослабляет параметры.

Комплементарность между разными системами проявляется на множестве функций и элементов. Она явно выделяется в связях и отношениях. На входах и выходах она выступает как фактор согласования. Последовательность действий также может характеризоваться комплементарностью или противоречивостью. В современных системах даже типы данных должны быть комплементарны технологиям обработки и алгоритмам [26].

Для современных информационных и вычислительных систем важным фактором является оценка качества системы. Основой оценки качества программного обеспечения в настоящее время являются два стандарта: отечественный стандарт ГОСТРИСО/МЭК25010-2015 «Информационные технологии (ИТ). Системная и программная инженерия. Требования и оценка качества систем и программногоо беспечения (SQuaRE). Модели качества систем и программных продуктов» и зарубежный стандарт ISO/IEC25010:2011 «Системная и программная инженерия. Требования и оценка качества систем и программного обеспечения (SQuaRE). Модели качества систем и программного обеспечения» [26]. По существу эти стандарты задают нормы компле- 
ментарности между программным обеспечением, данными и технологией обработки.

Комплементарность не всегда означает полное соответствие по всем параметрам системы. Она означать также либо частичное соответствие, либо согласованность только по ключевым показателям. Видов комплементарности в системах может быть много. Комплементарность может быть по функции, по цели, по входам выходам, по семантическому содержанию, по последовательности действий, по данным.

Параметр $F$ в выражении (5) задает системно-функциональную комплементарность систем или процессов. Системная функциональная комплементарность включает три вида: функциональную комплементарность (functional conformity), функциональное дополнение (functional complement), функциональную согласованность (functional consistency).

Функциональная комплементарность означает дополняемость и согласованность функций разных систем при решении определенной задачи. Функциональное дополнение означает, что функции разных систем дополняют друг друга при решении общей задачи. Например, система сбора информации, система первичной обработки, система моделирования, система обработки и система представления информации - комплементарны. Они действуют по схеме, показанной на рис. 2. Выход одной системы является входом для другой. Как правило, такая комплементарность свойственна специализированным системам или процессам. Функциональная согласованность означает непротиворечивость функций разных систем. В сравнении с функциональной комплементарностью это более слабая комплементарность.

Может существовать комплементарность по данным. Если существует система $A$ с набором данных $D A$ и система $B$ с набором данных $D B$, то комплементарность по данным означает

$$
D A \subset D B \oplus D B \subset D A .
$$

Выражение (6) показывает, что в случае комплементарности данные одной системы можно использовать для другой системы, но не наоборот. В выражении (6) комплементарность выступает как отношение принадлежности.

Параметр Sem задает семантическую комплементарность систем или моделей, которая включает три вида семантической комплементарности: семантическое соответствие, семантическое дополнение.

Семантическое соответствие как комплементарное отношение означает, что результаты процессов или функций семантически выражают одно и то же. Они подтверждают друг друга и не противоречат друг другу. Семантическое дополнение как комплементарное отношение означает, что результаты процессов или функций семантически дополняют друг друга и создают общую целостность при интерпретации результатов обработки. Комплементарность «семантическое соответствие» служит подтверждением результатов обработки. Комплементарность «семантическое дополнение» служит основой целостности результатов обработки. 
В математике существуют разные виды комплементарности, что дает основание выражать их разными описаниями. Например, для операций коммутативности

$$
(a+b)=(b+a) \forall a, b \in A
$$

и ассоциативности

$$
(a+b)+c=a+(b+c) \forall a, b \in A
$$

существует информационное соответствие и одновременно комплементарность. Для операций дистрибутивности

$$
\begin{gathered}
x f(a+b)=x f(b)+x f(c) \forall a, b \in A, \\
P_{1}=x f(a+b), P_{2}=x f(a)+x f(b), Q=f(a+b) .
\end{gathered}
$$

Существует информационное соответствие между $P_{1}, P_{2}$ и комплементарность между $Q$ и $P_{1}$.

\section{4. КОМПЛЕМЕНТАРНОСТЬ В ФУНКЦИОНАЛЬНЫХ ОПИСАНИЯХ}

Если рассматривать суперпозицию функций со значением истина, то ее наличие также является носителем функциональной комплементарности. В теории множеств комплементарность отражается отношениями принадлежности $\in$ или включения $\subseteq$. В функциональном анализе комплементарность выражается функциональной зависимостью.

Комплементарность в функциональных описаниях и схемах принятия решений может быть рассмотрена как композиция функций, отраженная в композиции формул. Формулы, включая логические, можно рассматривать как формализацию композиции функций. Различные формы функционального описания дают возможность проводить многосторонний анализ комплементарности и поиск ее разных форм

Функции отображают по-разному. Классическая запись $y=f(x)$ говорит о том, что функция преобразует входные переменные $x$ в выходные переменные $y$. Системная запись этого же процесса [28] $f: X \rightarrow Y$ говорит о том, что имеет место функциональное преобразование входного множества $X$ в выходное множество $Y$. Простая суперпозиция имеет вид

$$
g(f(x)) .
$$

Если функция $f$ выполнима и истинна, это влечет выполнение функции $g$ (рис. 2). Если функция $f$ выполнима и истинна и функция $g$ выполнима и истинна, то функции $g$ и $f$-комплементарны. Если функция $f$ или функция $g$ невыполнимы, то функции $g$ и $f$-некомплементарны. Выражение (7) имеет графическое представление. Оно показано на рис. 2. 


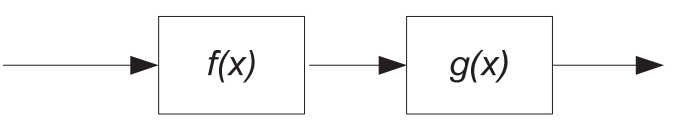

Puc. 2. Графическая форма суперпозиции двух функций

Fig. 2. Graphic superposition of two functions

Выражение (5) описывает суперпозицию функций $f(x)$ и $g(x)$. Схема на рис. 1 является функционально-логической, и ее основное назначение - проверка композиции функций $f(x)$ и $g(x)$. На рис. 1 выход $f(x)$ является входом для $g(x)$. Это означает комплементарность функций по выходу / входу. Функций может быть много. Конъюнктивная форма является основой комплементарных функций

$$
S=f_{1} \wedge f_{2}, \wedge \wedge f_{n} .
$$

В выражении (8) сложная функция, которая сформирована как конъюнкция более простых функций. Если хоть одна из функций $f_{n}$ невыполнима, то невыполнима $S$. Это является особенностью комплементарных функций функциональным дополнением. Комплементарность сложных функций оценивается по трем критериям: потоковая (вход / выход), функциональная (соответствие функций), логическая.

Если рассматривать схему как совокупность булевых функций, можно упростить анализ. В общем случае имеется $n$ булевых (принимающих значения 0 и 1) переменных $x_{1}, \ldots, x_{n}$, называемых входами. Также имеется некоторое число булевых переменных $y_{1}, \ldots, y_{m}$, называемых выходами или проводниками (рис. 1). Входы и выходы связывают функции. Для каждого проводника схемы может быть задана булева функция из набора булевых функций $B$, выражающая его через другие проводники и входы. Для обеспечения комплементарности требуется, чтобы не было циклов. При этом возможны два варианта. Булева функция является технической функцией и реализует работу с двоичными сигналами - это случай соответствия. Булева функция отражает логику других функций - это случай отражения. Проведенный анализ позволяет ввести понятие логической комплементарности. Логическая комплементарность, или логическая непротиворечивость, есть отношение внутри сложной системы, алгоритма или схемы принятия решений, для которой отсутствует логическая противоречивость в выполнении процессов или вычислений.

\section{ЗАКЛЮЧЕНИЕ}

Комплементарность является сложным видом отношений, описание которых в ряде случаев не осуществляется одним математическим или логическим оператором. Комплементарность можно рассматривать как группу специфических отношений, обобщающую более простые отношения. Комплементарность как характеристика является важной для сложных систем и вы- 
числительных процессов. Комплементарность повышает качество и надежность. Логическая комплементарность как отношение является важной составляющей схемы обработки информации или системы принятия решений. Наличие отношения комплементарности позволяет решать две задачи. Первая задача структурного анализа - задача анализа структуры системы или технологии на предмет согласованности и непротиворечивости. Вторая задача процессуального анализа - задача анализа процессов внутри структуры системы или технологии на предмет выполнимости и непротиворечивости. Можно констатировать, что на блоки обработки информации или функциональной системы можно составить разные виды описаний: логическое, системное и функционально логическое. Каждое описание допускает свой вид комплементарности и дает возможность оценить совокупную комплементарность. Для определения структуры необходимо использовать логические связки и функционально логическое описание. Логическая комплементарность доказывается и проверяется средствами математической логики. Функциональная комплементарность проверяется средствами функционального анализа. Системная комплементарность оценивается методами системного анализа.

\section{СПИСОК ЛИТЕРАТУРЫ}

1. Cottle R.W. Linear complementarity problem // Encyclopedia of Optimization. - Boston, MA: Springer, 2008. - P. 1873-1878.

2. Complementarity [Electronic resource]. - URL: https:/en.oxforddictionaries.com/definition/ complementarity (accessed: 19.06.2019).

3. Complementarity [Electronic resource]. - URL: https://www.merriam-webster.com/ dictionary/complementarity (accessed: 19.06.2019).

4. Tom Xu K., Farrell T.W. The complementarity and substitution between unconventional and mainstream medicine among racial and ethnic groups in the United States // Health Services Research. - 2007. - Vol. 42, N 2. - P. 811-826.

5. The microRNA.org resource: targets and expression / D. Betel, M. Wilson, A. Gabow, D.S. Marks, C. Sander // Nucleic Acids Research. - 2008. - Vol. 36, suppl. 1. - P. D149-D153.

6. Vallurupalli P., Kay L.E. Complementarity of ensemble and single-molecule measures of protein motion: a relaxation dispersion NMR study of an enzyme complex // Proceedings of the National Academy of Sciences. - 2006. - Vol. 103, N 32. - P. 11910-11915.

7. Kazempour S.J., Conejo A.J., Ruiz C. Strategic generation investment using a complementarity approach // IEEE Transactions on Power Systems. - 2011. - Vol. 26, N 2. - P. 940-948.

8. Богутдинов Б.Б., Цветков В.Я. Применение модели комплементарных ресурсов в инвестиционной деятельности // Вестник Мордовского университета. - 2014. - Т. 24, № 4. C. 103-116.

9. Brunello G. Labour market institutions and the complementarity between education and training in Europe // Education, training and labour market outcomes in Europe. - London: Palgrave Macmillan, 2004. - P. 188-210.

10. Xian W., Yuzeng L., Shaohua Z. Oligopolistic equilibrium analysis for electricity markets: a nonlinear complementarity approach // IEEE Transactions on Power Systems. - 2004. - Vol. 19, N 3. P. 1348-1355.

11. Цветков В.Я. Комплементарность информационных ресурсов // Международный журнал прикладных и фундаментальных исследований. - 2016. - № 2. - С. 182-185.

12. Isac G. Topological methods in complementarity theory. - Dordrecht: Springer Science \& Business Media, 2013. - 494 p. 
13. Fiske A.P. Complementarity theory: why human social capacities evolved to require cultural complements // Personality and Social Psychology Review. - 2000. - Vol. 4, N 1. - P. 76-94.

14. Garcia C.B. Some classes of matrices in linear complementarity theory // Mathematical Programming. - 1973. - Vol. 5, N 1. - P. 299-310.

15. Billups S.C., Murty K.G. Complementarity problems // Journal of Computational and Applied Mathematics. - 2000. - Vol. 124, N 1-2. - P. 303-318.

16. Верещагин Н.К., Шень А. Лекции по математической логике и теории алгоритмов. Ч. 2. Языки и исчисления. - 4-е изд., испр. - М.: МЦНМО, 2012. - 240 с.

17. Ben-Ari M. Mathematical logic for computer science. - 3rd ed. - London; New York: Springer, 2012. $-364 \mathrm{p}$.

18. Tsvetkov V.Ya. Not transitive method preferences // Journal of International Network Center for Fundamental and Applied Research. - 2015. - Vol. 3, iss. 1. - P. 34-42. - DOI: 10.13187/ jincfar.2015.3.34.

19. Бабурин Д.Е. Иерархический подход для автоматического размещения ациклических графов // Современные проблемы конструирования программ. - Новосибирск, 2002. - С. 7-37.

20. Tsvetkov V.Ya. Information situation and information position as a management tool // European Researcher. Series A. - 2012. - Vol. 12-1 (36). - P. 2166-2170.

21. Розенберг И.Н. Информационная ситуация как сложная система // Образовательные ресурсы и технологии. - 2017. - № 3 (20). - С. 69-77.

22. Ожерельева Т.А. Информационное соответствие и информационный морфизм в информационном поле // ИТНОУ: Информационные технологии в науке, образовании и управлении. -2017 . - № 4. - С. 86-92.

23. Номоконова О.Ю. Виды информационных соответствий // Славянский форум. -2018. № 2 (20). - C. 44-49.

24. Цветков В.Я. Решение проблем с использованием системного анализа // Перспективы науки и образования. - 2015. - № 1. - С. 50-55.

25. Кудж С.А. Системный подход // Славянский форум. - 2014. - № 1 (5). - С. 252-257.

26. Щенников А.Н. Комплементарность сложных вычислений // Славянский форум. 2018. - № 2 (20). - C. 118-123.

27. ISO/IEC 25010:2011. Systems and software engineering - Systems and software Quality Requirements and Evaluation (SQuaRE) - System and software quality models [Electronic resource]. URL: https://www.iso.org/standard/35733.html (accessed: 20.06.2019).

28. Месарович М., Такахара Н. Общая теория систем: математические основы. - М.: Мир, 1978. $-311 \mathrm{c}$.

Цветков Виктор Яковлевич, доктор технических наук, профессор Научно-исследовательского и проектно-конструкторского института информатизации, автоматизации и связи на железнодорожном транспорте (НИИАС), заместитель руководителя Центра стратегического анализа и развития. Основное направление научных исследований: геоинформатика, управление, информационные технологии. Автор свыше 600 научных трудов. Подготовлено 6 учебников, 41 монография и 40 брошюр. E-mail: cvj2@mail.ru

Tsvetkov Viktor Yakovlevich, Doctor of Technical Sciences, Professor in the Scientific Research and Design Institute for Information, Automation and Communication in Railway Transport (NIIAS), Deputy Head of the Center for Strategic Analysis and Development. The main direction of scientific research include geoinformatics, management, and information technology. He is the author of over 600 scientific papers including 6 textbooks, 41 monographs and 40 brochures. E-mail: cvj2@mail.ru 
DOI: $10.17212 / 1814-1196-2019-2-101-114$

\title{
Complementary relationships*
}

\author{
V.Ya. TSVETKOV
}

Research and Design Institute of Design Information, Automation and Communication on Railway Transport 27, Nizhegorodskaya Street, bldg 1, Moskow, 109029, Russian Federation

cvj2@mail.ru

\section{Abstract}

The organization of computation requires that computational processes have the properties of consistency and consistency. These properties are the result of complementarity and the presence of complementary relations. In the system theory, complex systems must have the properties of integrity and completeness. Integrity and completeness is a sign of complementary relations. In Earth sciences, in particular, in the field of geoinformatics, a set of sequential technologies for processing spatial information is used. Information processing is preceded by information gathering technology. Then the technology of preprocessing and rejection follows. The next steps are technology modeling, storage, and so on. A set of different technologies that solve a common problem, connect complementary relationships. The article explores complementary relationships as a specific kind of complex relationships. The complexity of complementary relationships is due to the diversity of their forms. Complementary relationships are present in communication networks, in information situations, and in complex systems. Complementary relationships create a complement property or a state of complementarity. The article systematizes the types of complementarity and introduces the following concepts: functional complementarity, systemic complementarity, and logical complementarity. Logical complementarity complements other types of complementarity. It allows you to test various technologies and structures for inconsistency and truth. Logical complementarity allows checking various processes for truth and non-inconsistency. Program verification methods use the complementarity property.

Keywords: complementarity, relationships, informational relationships, complementary relationships, consistency

\section{REFERENCES}

1. Cottle R.W. Linear complementarity problem. Encyclopedia of Optimization. Boston, MA, Springer, 2008, pp. 1873-1878.

2. Complementarity. Available at: https://en.oxforddictionaries.com/definition/complementarity (accessed 19.06.2019).

3. Complementarity. Available at: https://www.merriam-webster.com/dictionary/complementarity data view (accessed 19.06.2019).

4. Tom Xu K., Farrell T.W. The complementarity and substitution between unconventional and mainstream medicine among racial and ethnic groups in the United States. Health Services Research, 2007, vol. 42, no. 2, pp. 811-826.

5. Betel D., Wilson M., Gabow A., Marks D.S., Sander C. The microRNA.org resource: targets and expression. Nucleic Acids Research, 2008, vol. 36, suppl. 1, pp. D149-D153.

6. Vallurupalli P., Kay L.E. Complementarity of ensemble and single-molecule measures of protein motion: a relaxation dispersion NMR study of an enzyme complex. Proceedings of the National Academy of Sciences, 2006, vol. 103, no. 32, pp. 11910-11915.

\footnotetext{
* Received 13 December 2018.
} 
7. Kazempour S.J., Conejo A.J., Ruiz C. Strategic generation investment using a complementarity approach. IEEE Transactions on Power Systems, 2011, vol. 26, no. 2, pp. 940-948.

8. Bogutdinov B.B., Tsvetkov V.Ya. Primenenie modeli komplementarnykh resursov v investitsionnoi deyatel'nosti [Application of the model of complementary resources in investing activities]. Vestnik Mordovskogo universiteta - Mordovia University Bulletin, 2014, vol. 24, no. 4, pp. 103-116.

9. Brunello G. Labour market institutions and the complementarity between education and training in Europe. Education, training and labour market outcomes in Europe. London, Palgrave Macmillan, 2004, pp. 188-210.

10. Xian W., Yuzeng L., Shaohua Z. Oligopolistic equilibrium analysis for electricity markets: a nonlinear complementarity approach. IEEE Transactions on Power Systems, 2004, vol. 19, no. 3, pp. 1348-1355.

11. Tsvetkov V.Ya. Komplementarnost' informatsionnykh resursov [Complementarity information resources]. Mezhdunarodnyi zhurnal prikladnykh i fundamental'nykh issledovanii - International journal of applied and fundamental research, 2016, no. 2, pp. 182-185.

12. Isac G. Topological methods in complementarity theory. Dordrecht, Springer Science \& Business Media, 2013. 494 p.

13. Fiske A.P. Complementarity theory: why human social capacities evolved to require cultural complements. Personality and Social Psychology Review, 2000, vol. 4, no. 1, pp. 76-94.

14. Garcia C.B. Some classes of matrices in linear complementarity theory. Mathematical Programming, 1973, vol. 5, no. 1, pp. 299-310.

15. Billups S.C., Murty K.G. Complementarity problems. Journal of Computational and Applied Mathematics, 2000, vol. 124, no. 1-2, pp. 303-318.

16. Vereshchagin N.K., Shen' A. Lektsii po matematicheskoi logike i teorii algoritmov. Ch. 2. Yazyki i ischisleniya [Lectures in mathematical logic and computability theory. Pt. 2. Languages and Calculi]. 4th ed. Moscow, MCCME Publ., 2012. 240 p.

17. Ben-Ari M. Mathematical logic for computer science. 3rd ed. London, New York, Springer, 2012. $364 \mathrm{p}$

18. Tsvetkov V.Ya. Not transitive method preferences. Journal of International Network Center for Fundamental and Applied Research, 2015, vol. 3, iss. 1, pp. 34-42. DOI: 10.13187/jincfar.2015.3.34.

19. Baburin D.E. Ierarkhicheskii podkhod dlya avtomaticheskogo razmeshcheniya atsiklicheskikh grafov [Hierarchical approach to automatic placement directed acyclic graphs]. Sovremennye problemy konstruirovaniya program [Modern problems of program construction]. Novosibirsk, 2002, pp. 7-37.

20. Tsvetkov V.Ya. Information situation and information position as a management tool. European researcher. Series A, 2012, vol. 36 (12-10, pp. 2166-2170.

21. Rozenberg I.N. Informatsionnaya situatsiya kak slozhnaya sistema [Information situation as complex system]. Obrazovatel'nye resursy i tekhnologii - Educational Resources and Technologies, 2017 , no. 3 (20), pp. 69-77.

22. Ozherel'eva T.A. Informacionnoe sootvetstvie i informacionnyj morfizm v informacionnom pole [Information conformity and informational morphism in the information field]. ITNOU: Informatsionnye tekhnologii v nauke, obrazovanii i upravlenii, 2017, no. 4, pp. 86-92. (In Russian).

23. Nomokonova O.Yu. Vidy informatsionnykh sootvetstvii [Types of information correspondences]. Slavyanskii forum - Slavic Forum, 2018, no. 2 (20), pp. 44-49.

24. Tsvetkov V.Ya. Reshenie problem s ispol'zovaniem sistemnogo analiza [Solving problems using a systematic analysis]. Perspektivy nauki i obrazovaniya - Perspectives of Science and Education, 2015, no. 1, pp. 50-55.

25. Kudzh S.A. Sistemnyi podkhod [Systems approach]. Slavyanskii forum - Slavic Forum, 2014, no. 1 (5), pp. 252-257.

26. Shchennikov A.N. Komplimentarnost' slozhnykh vychislenii [Complementarity of complex computations]. Slavyanskii forum - Slavic Forum, 2018, no. 2 (20), pp. 118-123. 
27. ISO/IEC 25010:2011. Systems and software engineering - Systems and software Quality Requirements and Evaluation (SQuaRE) - System and software quality models. Available at: https://www.iso.org/standard/35733.html (accessed 20.06.2019).

28. Mesarovic M.D., Takahara Y. General systems theory: mathematical foundations. New York, Academic Press, 1975 (Russ. ed.: Mesarovich M., Takakhara N. Obshchaya teoriya sistem: matematicheskie osnovy. Moscow, Mir Publ., 1978. 311 p.).

Для цитирования:

Цветков В.Я. Комплементарные отношения // Научный вестник НГТУ. - 2019. № 2 (75). - C. 101-114. - DOI: 10.17212/1814-1196-2019-2-101-114.

For citation:

Tsvetkov V.Ya. Komplementarnye otnosheniya [Complementary relationships]. Nauchnyi vestnik Novosibirskogo gosudarstvennogo tekhnicheskogo universiteta - Science bulletin of the Novosibirsk state technical university, 2019, no. 2 (75), pp. 101-114. DOI: 10.17212/1814-1196-2019-2101-114.

ISSN 1814-1196, http://journals.nstu.ru/vestnik Science Bulletin of the NSTU Vol. 75, No 2, 2019, pp. 101-114 